\title{
Application of Internet of Things Technology Based on Artificial Intelligence in Electronic Information Engineering
}

\author{
Haibo Zhang ${ }^{1}$ and Sining Cheng $\mathbb{D}^{2}$ \\ ${ }^{1}$ School of Electronics and Information Engineering, Nanchong Vocational and Technical College, Nanchong 637131, \\ Sichuan, China \\ ${ }^{2}$ Tengjing Network Academy, Haikou University of Economics, Haikou 571127, Hainan, China \\ Correspondence should be addressed to Sining Cheng; siningcheng@163.com
}

Received 25 August 2021; Revised 24 November 2021; Accepted 2 December 2021; Published 31 January 2022

Academic Editor: Sang-Bing Tsai

Copyright (c) 2022 Haibo Zhang and Sining Cheng. This is an open access article distributed under the Creative Commons Attribution License, which permits unrestricted use, distribution, and reproduction in any medium, provided the original work is properly cited.

\begin{abstract}
Artificial intelligence is an intelligent system that can perform tasks in a complex and changeable environment without special manual intervention. It is a technology that can continuously optimize decisions and behaviors through machine learning. It is a portrayal of human intelligence in computers. The simple explanation is usually "artificial intelligence." Since the invention of artificial intelligence, theory and technology have been continuously developed and its scope has also been continuously expanded. With the improvement of people's rich and colorful material living standards, more and more areas of life where artificial intelligence products are used can be widely and conveniently accessed by people, but the abuse of artificial intelligence technology will also become the biggest public safety in the future. Artificial intelligence technology is used by some terrorists, making it more convenient for terrorist attacks. To this end, this article aims to study the artificial intelligence Internet of Things technology in electronic information engineering. It is hoped that the artificial intelligence Internet of Things technology can be better optimized and the technical means can be mastered and rationally used to improve people's material living conditions and happiness. This article proposes how to better apply the artificial intelligence Internet of Things technology to electronic information engineering and analyze optimization schemes and early warning models to better simulate life and optimize information conditions. The experimental results of this paper are analyzed through model construction. Finally, this paper selects and uses 16 standard mechanics datasets to find that it is beneficial to the better application of artificial intelligence Internet of Things technology, which verifies the accuracy and overall applicability of the method.
\end{abstract}

\section{Introduction}

With the continuous development of Internet information science and technology, many technologies are developing in a more intelligent direction. Artificial intelligence (AI) technology is an important means to build the computing space of the Internet of Things. It belongs to a marginal field, a natural science. Scientists are hoping to understand the nature of intelligence through research and understanding and strive to create a new type of intelligent machinery that can respond to it in various forms and ways similar to human intelligence. Research in this field involves robotic engineering, language recognition, image recognition, natural language processing, and expert systems. Since the birth of artificial intelligence, the development of theory and technology has become more mature and the field of application has been continuously expanded. In the near future, the high-tech products that artificial intelligence bring to the future will be regarded as a vivid expression of human wisdom. With the development of information technology, especially the rapid development of cloud computing and other technologies, artificial intelligence (AI) technology has been developed by leaps and bounds. The emergence of new technological capabilities has made artificial intelligence surveillance a reality. Artificial intelligence surveillance electronic information engineering was born under this background, providing more reliable protection for the safety of human lives and reducing or avoiding tragedies. 
In the twenty-first century, the rapid development of electronic information technology has brought tremendous changes to people's lives. Among them, logistics management based on electronic information technology is developing rapidly and its research is particularly important.

As the electronic information technology industry is gradually playing an increasingly important role, it has become an important growth point for enhancing its economic competitiveness on a global scale. Our country's decision to take a new path to modernization and industrialization in recent years demonstrates the progress of our country's electronic information industry. Electronic information products have the typical advantages of short market life cycle and time, frequent product changes, and focus on networked applications. Innovation activities are derived from two guidelines: technological progress and user needs. We must use technological innovation and improve product value. The introduction of artificial intelligence technology and the Internet of Things in the process of promoting electronic information technology and product innovation plays a very important role in the development of the country's electronic information industry and the enhancement of market competitiveness.

The field of artificial intelligence (AI) has a long and intertwined history. Hassabis et al. believed that a better understanding of biological brains can play a vital role in building intelligent machines. They investigated the historical interaction between $\mathrm{AI}$ and the field of neuroscience and highlighted the current advances in AI inspired by neural computing research in humans and other animals. Finally, it emphasizes common themes that may be crucial to advancing future research in this field, but only a general scope direction is determined and the practicality is not very high [1]. In the construction of a digital museum, it is very difficult to manage so many collections and find a place to place a collection. Zhao-Hui proposed a technology to identify the collection; this technology is information technology, and it created the foundation for the smart museum. However, the persuasive power of this technology is not strong [2]. Ogidiaka has been creating problems with psychological factors. In this case, the intelligent model is related to rational statistics and economic concepts. Simply put, it is the ability to make correct decisions, plans, or conclusions. The adoption of random representation and statistical learning methods has led to a higher degree of integration and intersection between artificial intelligence, machine learning, statistics, cybernetics, neuroscience, and other disciplines. However, the current technology is not mature enough and there are some shortcomings [3].

From the human beings to the intelligent era, the convenient application of intelligent technology makes human social life and work more convenient. There are also huge security risks [4]. Therefore, scientific analysis of the risks of artificial intelligence technology and standardized use of artificial intelligence electronic information technology will bring opportunities for the future sustainable development of mankind and reduce harm and loss. This article uses the combination of artificial intelligence Internet of Things technology and electronic information engineering to explore the application of artificial intelligence Internet of Things technology in electronic information equipment. It uses the artificial firefly algorithm and ant colony algorithm to explore the optimization of the formula to find the minimum value of the shortest path. Indepth study of the relationship between artificial intelligence Internet of Things technology and electronic information engineering through SOM neural network early warning model construction, agent behavior tree construction, SVR prediction model analysis and optimization, and neural network early warning forecast model analysis strengthens electronic information engineering and artificial intelligence. The synergy of networking is conducive to the research, exploration, and optimization of the application of artificial intelligence Internet of Things technology in electronic information engineering.

\section{The Method of Electronic Information Engineering Based on Artificial Intelligence Internet of Things Technology}

\subsection{Internet of Things Technology}

2.1.1. Smart Home in the Internet of Things Environment. The specific layers of the Internet of Things are the perception layer, the network layer, and the application layer. A corresponding smart home system security framework is proposed, namely, node security, information transmission security, and information use security, and a security mechanism is set up at each layer of the transmission. The security system structure of the Internet of Things is constructed, and its framework is shown in Figure 1.

2.1.2. Internet of Things Gateway. In the perception layer of the system, there are a large number of sensors. These sensors form a sensor network for data detection. The sensor data are transmitted to the wide area network through the sensor network at the network level [5]. The gateway achieves the purpose of interconnecting wide area information by providing protocol conversion between the perception layer and the wide area network. Therefore, it has an indispensable position in the network layer [6]. Figure 2 shows the status of the Internet of Things in the system: the gateway is connected to the server through the port, and the message is processed through the HTTP protocol; the serial port is connected to the underlying coordinator to receive and forward system data; to achieve the conversion of Modbus/ TCP and Modbus/RTU protocols. In terms of function, the gateway mainly realizes the data duplex communication function and network organization ability; after physically connecting to ZigBee and the coordinator, it monitors the terminal equipment of the system in real time, by connecting modem equipment (GPRS module), cellular mobile communication equipment ( $3 \mathrm{G}$ module), wired and WiFi to transmit the data sensed by the sensor network to the server; connect the SD analog camera to collect on-site pictures regularly and store and forward the pictures on the SD card; display the data sensed by the sensor network through the 


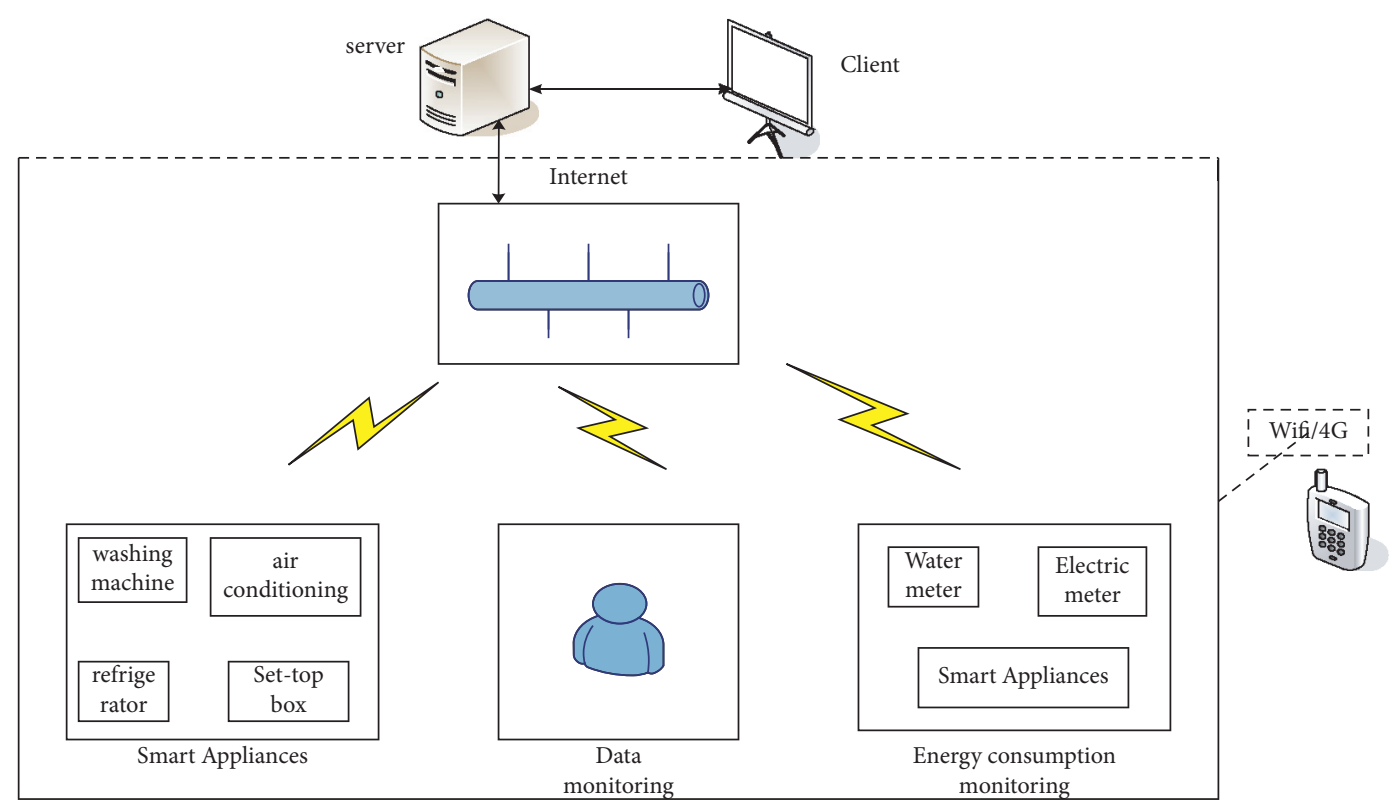

FIgURE 1: Structure diagram of the smart home control system.

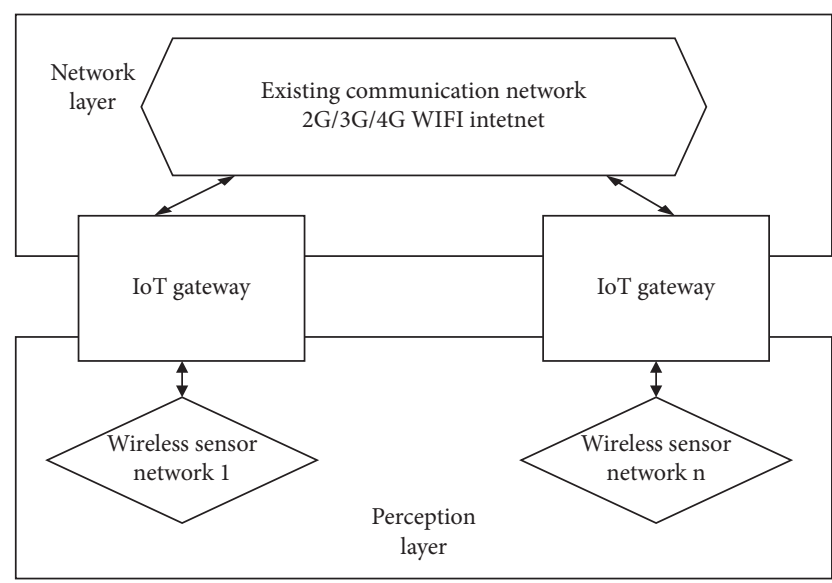

Figure 2: IoT gateway.

touch screen, and the camera collects the picture and the working status of the gateway; remotely login to the server to view the system data, pictures, and the working status of the gateway [7].

2.2. Artificial Intelligence. Affected by many factors, it is difficult for artificial intelligence to truly exhaust the mysteries of human intelligence. The academic world cannot form a unified definition of artificial intelligence [8].

Advantages: AI technology has enriched people's lives and provided great help to people's lives. The combination of artificial intelligence technology and various tools and materials of human life has completely changed people's traditional lifestyles and added new colors to people's lives. Artificial intelligence enriches people's lives and makes people's lives smarter [9].

Disadvantages: the development of AI has leaked people's privacy [10].
Artificial intelligence algorithms: there are many artificial intelligence algorithms applied to network reconstruction. The widely used human king neural network, simulated annealing method, genetic algorithm, particle swarm algorithm, expert system, etc. are briefly introduced below [11].

Simulated annealing method: a method that selects an appropriate convergence index according to the difference in temperature to assist in accelerating the speed of the SA algorithm [12].

Particle swarm algorithm: using binary coding, the PSO algorithm is used to reconstruct the problem. The improvement of PSO algorithm also has many applications in reconstruction.

Electronic information has developed rapidly in recent years, and global competition has intensified. It has become one of the most important strategic industries in the world. In order to reach the peak of industrial competition, countries have pushed the electronic information industry to the top of their national strategies. In the past ten years, our country's electronic information industry has been developing very well, its strength has continued to increase, the proportion of output value in GDP has continued to increase, the driving force for economic growth has continued to increase, and its impact on economic growth has also continued to increase. More and more attention has been paid to the development of electronic information manufacturing industry.

The characteristics of the electronic information industry: (1) parallel growth and benefits: along with the increase in output, there will be a corresponding increase in efficiency; (2) large demand for technology and investment: because electronic information products are updated quickly and have high requirements for equipment and technology, a large amount of capital and technical investment are required to keep up with the speed of product upgrades; (3) clustering and relevance to other industries: the output of the 
electronic information industry is an indispensable input for related industries [13].

Our country's modern electronic information industry has increasingly become the leading and pillar industry in the mainland of our motherland, promoting our country's social and economic development. Figure 3 shows the change in the total output value of the domestic electronic information technology industry.

As shown in Figure 4, we can find that, from 1997 to 2000 , the domestic electronic information industry developed at a high speed higher than the growth rate of the manufacturing industry. It experienced the startup and growth stages and entered the mature stage after the twentyfirst century, and the growth rate began to lag behind because of the growth rate of the manufacturing industry.

2.3. BP Network Model Construction. This article uses MATLAB software to create a BP network prediction model and conducts learning training and system prediction analysis on the established system model. The construction steps of the BP neural network model are shown in Figure 5:

(1) Through the determination of the number of layers of the BP neural network, the transfer function, the number of neurons in each layer, and the assignment of weights and thresholds are selected; then, the system structure design is carried out [14]

(2) Ben selects the initial model, establishes appropriate training learning and rule functions, sets relevant training parameters, etc., and then trains through the model

(3) Use the established model to predict the processed data to be tested and analyze the prediction results

2.4. Artificial Firefly Algorithm. In the original firefly algorithm, some fireflies randomly scattered them in the troubleshooting area during initialization. The fluorescein content of these fireflies is basically the same at startup. Each firefly should be unique. The fireflies communicate with other fireflies within their main decision-making range, so as to be able to achieve control of other fireflies distributed in the surrounding area. The purpose of the event: the fluorescein value of each firefly is determined by the distance between the position of the firefly and the optimal position of the solution. The closer to the optimal position of the solution, the higher the fluorescein content of the firefly. The communication mechanism of fireflies is as follows: all fireflies are in the collection of other fireflies, the brighter the tail, the higher the fluorescein content, the stronger the ability to attract and approach the same firefly, and the other fireflies will spontaneously change to the fluorescein content and move in the direction of the taller fireflies [15].

In the optimization phase of the firefly algorithm, each iteration consists of the fluorescein update phase, the neighbor determination phase, the movement probability update phase, the position update phase, and the sensing radius update phase. Let $f(x)$ be the fitness evaluation function, and $C_{\mathrm{i}}(n)$ represents the fluorescein concentration

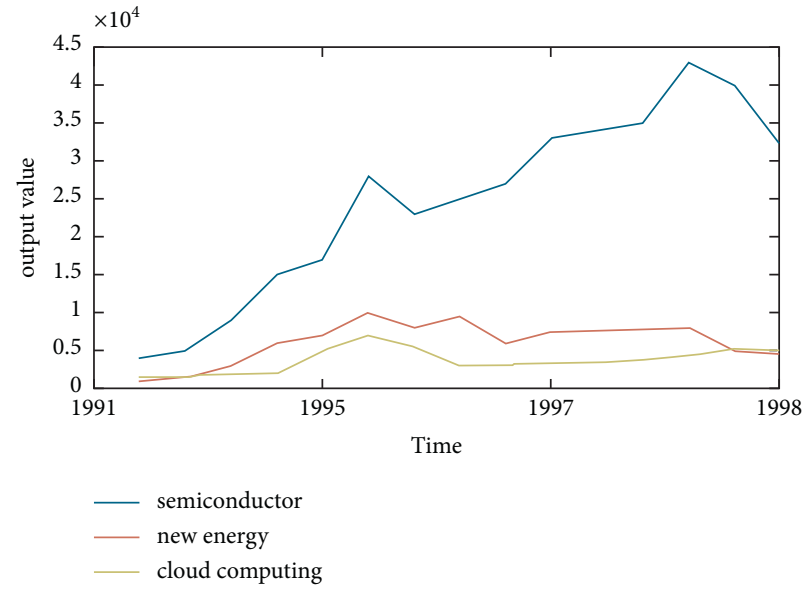

Figure 3: Changes in the output value of the domestic electronic information industry.

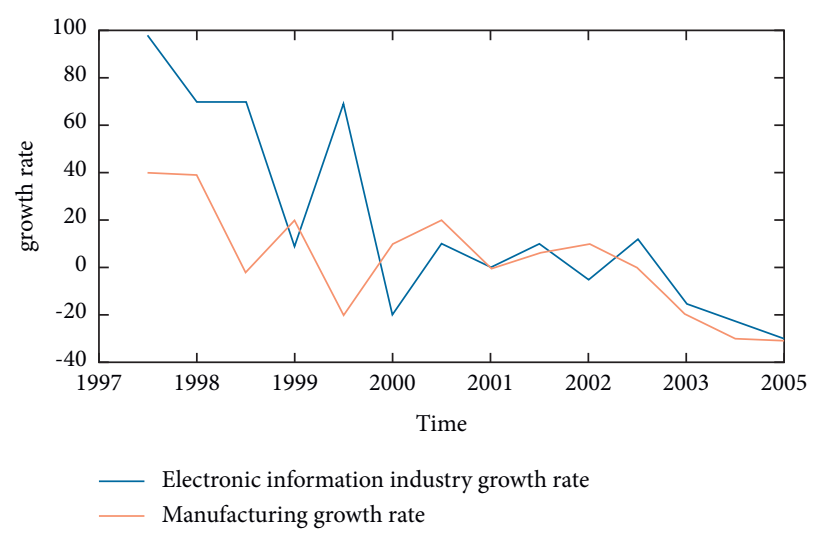

FIGURE 4: Growth rate of the domestic electronic information industry.

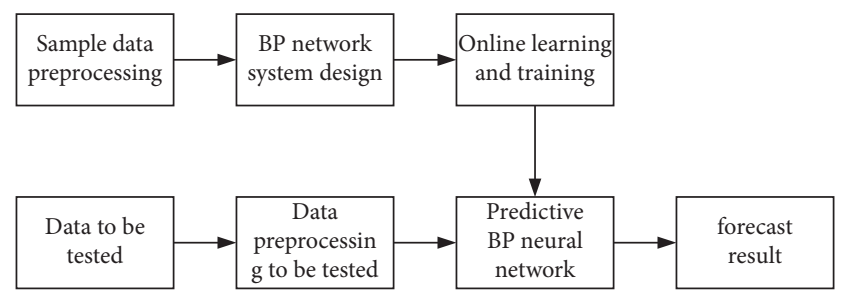

FIGURE 5: BP neural network model establishment steps.

of the $i$-th firefly in the Nth iteration, and the four stages are described as follows.

Initialization stage: first, NP fireflies are randomly generated in the search area, $x_{i}, g=\left[x 1, x 2, \ldots, x_{m}\right]$, where $g$ represents the number of iterations, $i=1,2, . ., \mathrm{NP}$; all fireflies in the initialization stage have fluorescein concentration $\operatorname{li}(g)=0$. At the same time, initialize the fluorescein volatilization coefficient $\rho$ of the firefly algorithm, the fitness extraction ratio $\gamma$, the step size step, the neighborhood change rate $\beta$, the decision radius $r d$, the neighbor number threshold gt, and the maximum number of iterations iter_max [16]. 
Fluorescein renewal stage: each firefly updates its fluorescein value according to the current firefly fitness value $f\left(x_{i}\right)$, that is, the fluorescein value at this time is the fluorescein value at the previous moment minus the fluorescein value volatilized over time plus its fitness value of a certain proportion at this time. The specific update rules are expressed as follows:

$$
C_{i}(n)=(1-\rho) * C_{i}(n-1)+\alpha * f\left(x_{i}(n)\right),
$$

where $\rho$ is the fluorescein volatilization coefficient and $\alpha$ is the fitness extraction ratio.

Determine the neighbor stage: each firefly looks for its neighbors within the decision radius, and its neighbor set is composed of neighbors that meet the following conditions:

$$
g_{t}=\left\{a: C_{i}(t)<C_{a}(t) \& \& d(i, a)<r_{d}(i) r_{d}(i)\right\} .
$$

In formula (2), $d(i, a)$ represents the Euclidean distance between the $i$-th and $a$-th firefly.

Moving probability update order: the $i$-th firefly determines the probability of moving to the $j$-th firefly by comparing the fluorescence brightness values of the fireflies in its neighbor set. The calculation method is as follows:

$$
P_{i, a}=\frac{\left(C_{a}(g)-C_{a}(g)\right)}{\sum_{K \in N_{i(t)}} l_{k}(t)-l_{i}(t)} .
$$

Position update stage: the $i$-th firefly determines to move a small step to firefly $j$ according to the roulette method, the moving step is step, and its position update formula is as follows:

$$
x_{i}(g+1)=x_{i}(g)+\operatorname{step} * \frac{\left(x_{a}(g)-x_{i}(g)\right)}{\left\|x_{a}(g)-x_{i}(g)\right\|} .
$$

Sensing radius update stage: after the previous step is completed, the sensing radius needs to be updated accordingly. The specific update formula is as follows:

$\mathrm{r}_{d}^{i}(g+1)=\min \left\{r_{0}, \max \left\{0, r_{\mathrm{d}}^{i}(g)+\gamma *\left(n_{t}-\left|N_{i}(g)\right|\right)\right\}\right\}$.

The flowchart of the artificial firefly algorithm is shown in Figure 6.

2.5. Ant Colony Algorithm. It is an algorithm based on bionics based on the principle of ant foraging. Ant Colony Algorithm (ACA) was first proposed by Italian scholars Dorigo et al. Little ants can always find food and can find the shortest path from the nest to the food/destination. More importantly, the ants can dynamically choose the optimal path in a dynamically changing environment [17].

In order to improve the development of artificial intelligence and Internet of Things technology electronic information engineering construction, the following symbols are introduced:

$M$ represents the number of ants in the algorithm.

$N$ represents the number of cities/nodes in the algorithm.

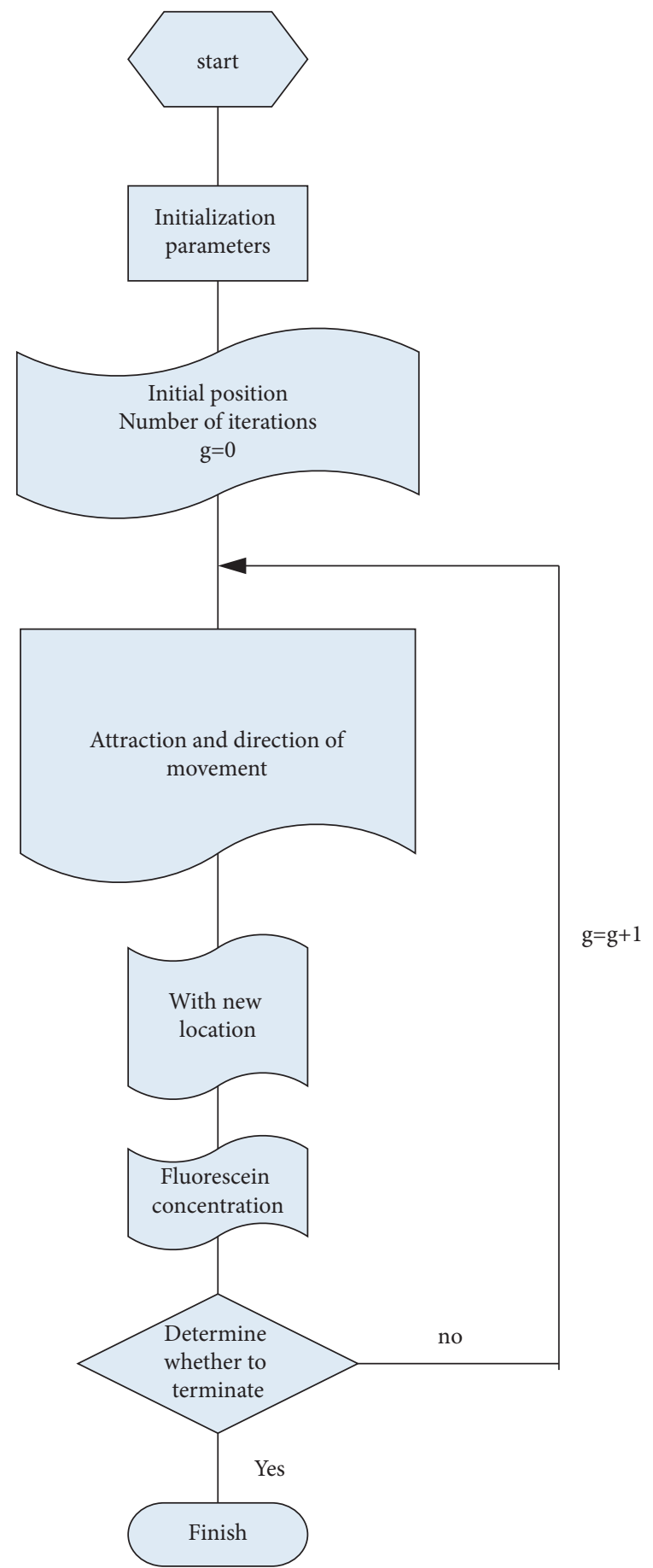

FIgURe 6: Flowchart of the artificial firefly algorithm.

$L$ represents the distance between city A and city B. $C_{\mathrm{i}}(\mathrm{t})$, at time $t$, represents the number of ants located in city $i$, where

$$
M=\sum_{\mathrm{a}=1}^{N} C_{i}(t)
$$

$\delta_{a b}(t)$, at time $t$, represents the pheromone on the city $(\mathrm{a}, \mathrm{b})$. 
At the beginning of the algorithm, the pheromone on each path is set to a constant C. During the movement, the probability of the ant selecting each optional node is calculated according to (7) and then a random number is generated according to the probability of each node, and you can roughly choose the city/node the ant visits next time.

$p_{a b}^{k}(t)$ represents the probability that ant $k$ chooses the path at node $i(a, b)$ :

$$
p_{a b}^{k}(t)=\left\{\begin{array}{l}
\frac{\delta_{a b}(t) \eta_{a b}^{\beta}}{\sum_{u \in t a b u_{k}} \delta_{a u}^{\alpha} \eta_{a u}^{\beta}} b \in t a b u_{k}, \\
0, b \in t_{a b u_{k}}
\end{array}\right.
$$

where $\eta_{\mathrm{ab}}$ represents the visibility of the path $(\mathrm{a}, b)$,

$$
\eta_{a b}=\frac{1}{d_{a b}}
$$

$\alpha$ represents the importance factor of pheromone, $\beta$ represents the importance factor of path visibility, and $N_{\mathrm{ab}}$ represents the number of algorithm iterations.

$t_{a b u}$ is the taboo table of ant $k$, which records the information of the cities that the ants have traveled. In the next route selection, the ants cannot choose the nodes in $t a b u_{k}$. At the same time, $t a b u_{k}$ also records the order information of the cities that the ants have traveled.

After $n$ times (there are $n$ cities in the algorithm), $m$ ants have completed an iterative process. According to the node information saved by $t a b u_{k}$, calculate the distance traveled by each ant Lat_k and calculate the shortest path Lat in this iteration at _min. In the ACA, the above process will be repeated $N_{\text {at }}$ times. After each iteration is completed, the pheromone on each path will be updated according to the ant's selection in $t_{a b u_{k}}$ to guide the ant's selection in the next iteration:

$$
\begin{aligned}
L_{a t-\min } & =\min \left(L_{a t-k}, k=1,2,3, \ldots, m\right), \\
\delta_{a b}(t+1) & =\rho \delta_{a b}(t) \Delta \delta_{a b}^{k}(t, t+1),
\end{aligned}
$$

where $\rho$ represents the pheromone persistence factor and $\rho-1$ represents the pheromone volatilization coefficient.

$$
\Delta \delta_{a b}(t, t+1)=\sum_{k=1}^{m} \Delta \delta_{a b}^{k}(t, t+1) .
$$

$D / L^{k}$ represents the path of ant $k(\mathrm{a}, \mathrm{b})$ :

$$
\Delta \delta_{a b}^{\mathrm{k}}(t, t+1)=\left\{\begin{array}{l}
\frac{D}{L^{k}} . \\
0
\end{array}\right.
$$

In formula (12), $\Delta \delta_{\mathrm{ab}}^{\mathrm{k}}(t, t+1)$ represents the contribution of the $k$-th ant to the pheromone on the path $(a, b)$ in the total path passed this time and $\Delta \delta_{\mathrm{ab}}(t, t+1)$ represents the path $(a, b)$ selected by the ant during this iteration. The variation of pheromone is shown in Equation (12). In equation (12), $D$ is a constant and $L^{\mathrm{k}}$ represents the total path length of the $k$-th ant through the cycle process. In ACA, the number of algorithm iterations is $N_{\mathrm{a} b}$. The shortest path is the minimum value of the shortest path found in each iteration:

$$
L_{\min }=\min L_{\mathrm{a} t-\min }\left(a t=1,2, \ldots, N_{a t}\right) .
$$

2.6. Summary of This Section. This section first introduces the smart home control system under the Internet of Things environment. On the basis of mastering the basic principles of the Internet of Things, it further describes the characteristics of the Internet of Things gateway, depicts the overall block diagram of the Internet of Things gateway system, provides a detailed introduction of artificial intelligence and its advantages and disadvantages, etc. The artificial intelligence algorithm, artificial firefly algorithm, artificial firefly algorithm flowchart, traffic control signal method, etc. are introduced, and the ant colony algorithm is analyzed in detail to determine its optimal path. The optimization and exploration of the formula for finding the minimum value of the shortest path further show that the artificial intelligence Internet of Things technology and electronic information engineering are closely related and promote each other.

\section{Experiments in Electronic Information Engineering Based on Artificial Intelligence and Internet of Things Technology}

3.1. Construction of Agent Behavior Tree. Commonly used AI decision models include state machines and behavior trees. The state machine is closer to human thinking, but the behavior tree is more intuitive and flexible. Therefore, the behavior tree has become the mainstream model of complex AI. The behavior tree can be regarded as a tree-type data structure, in which there are leaf nodes and composite nodes. At the same time, the behavior is divided into many levels, and lower-level behaviors can be combined to become higher-level behaviors. When the behavior tree needs to be updated, the structure will perform DFS operations, reaching the end leaf nodes in turn, and the leaf nodes will feed back different results according to different situations, for example, it can be "executed" or "nonexecuted." Because of such a tree structure, each branch is not traversed at the same time. At the same time, using this feature, you can easily set the priority level of behavior actions, allowing the front leaf nodes to take on more important behaviors [18].

Features: (1) the highly modular state removes the jump logic in the state, making the state itself a "behavior." (2) Behavior nodes and control nodes can reuse the same control nodes that can appear in different branches of the behavior tree. Increasing the types of control nodes, the same behavior nodes can be connected with different control nodes to make different AIs, so the purpose of multiplexing behaviors can also be achieved. There are many kinds of nodes in the behavior tree, and we can even customize the nodes to achieve the target function, as shown in Figure 7.

This behavior tree comprehensively uses the combination of selection nodes and sequence nodes and uses the 


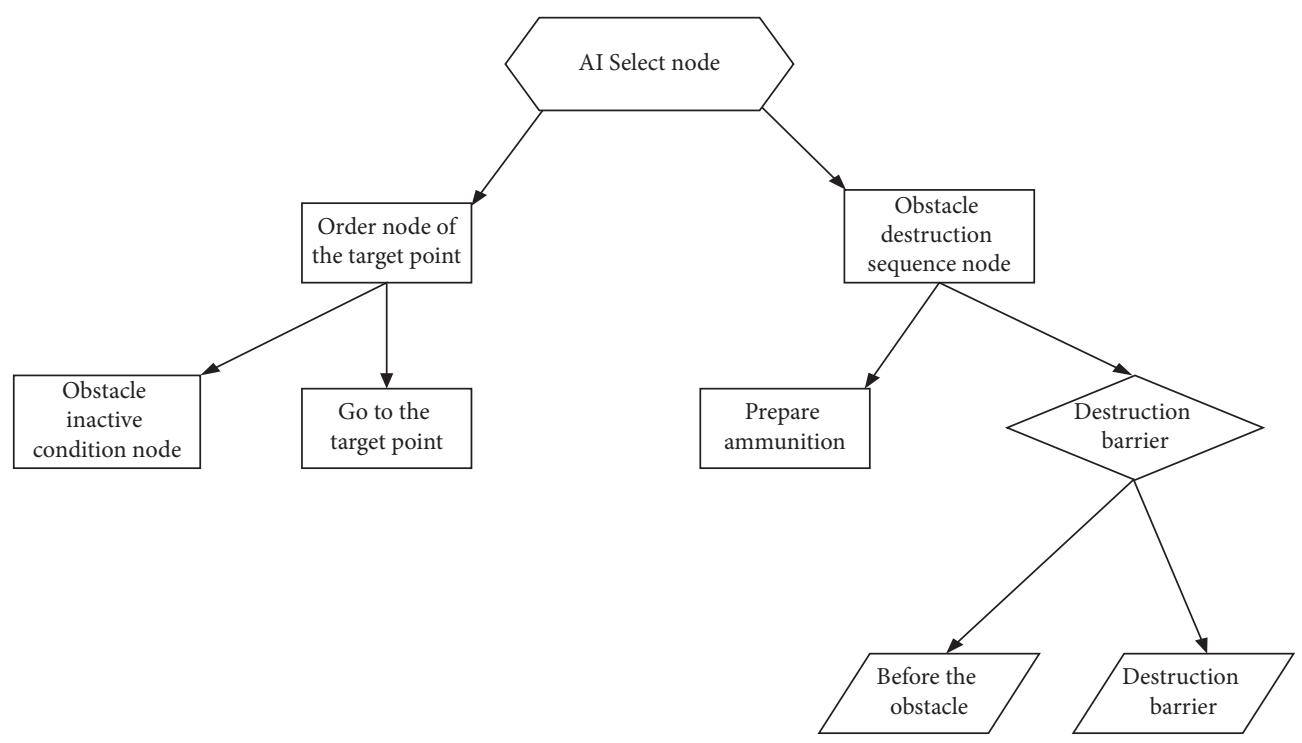

FIgURE 7: Common logical representation of behavior tree.

characteristics of the behavior tree to express the above logic more clearly, which can better realize the behavior of the agent [19].

\subsection{Construction of the Early Warning Model of SOM Neural} Network. The establishment of an early warning model based on neural networks can effectively monitor the impact of foreign products on our country's domestic industries and the possible harm caused by some potential damages. However, from the current situation of the electronic information industry, it is more important to prevent foreign countries from antidumping our country [20].

The structure flowchart of using neural network to generate early warning model is shown in Figure 8.

The early warning model generated by the neural network also has the ability to keep pace with the times. It can conveniently store and update the newly generated data, so that the result of the judgment is always associated with the international form. The block diagram of the specific operation is given in Figure 9.

Because the model designed in this paper has no special restriction rules for electronic information engineering in the selection of characteristic parameters, the general form is given, so that the model can be applied to the maximum expansion of artificial intelligence electronic information engineering, and the applicability of the model is further proved [20].

\subsection{Construction of the Production Function Model of the} Electronic Information Industry. We will analyze the various production factors invested in the development of the electronic information industry through the $\mathrm{C}$-D production function and study the relationship between the factors of various production sectors and economic growth. Because of the wide range of electronic information industry, we have improved and optimized the C-D production function for the convenience of calculation.
C-D production function:

$$
Y=A K^{\alpha} L^{\beta} .
$$

After introducing the technical variable $C^{i j}$, its production function is

$$
Y=A K^{\alpha} L^{\beta} C^{i j} .
$$

Due to the development of electronics, information technology engineering needs to study the growth of electronic information industry, and the introduction of external variable technology can clearly reflect the comprehensive information development index $C^{i j}$ of the development level of electronic information technology and the production level of the electronic information industry

From this, the production function used to study the specific industry of the electronic information industry is obtained:

$$
Y=A K^{\alpha} L^{\beta} C^{\mathrm{h} y} .
$$

Among them, $A$ is other technological progress factors, $C$ is the comprehensive development index of informatization, $h$ is the elasticity of the informatization index, $\alpha$ and $\beta$ are the output elasticities of capital and labor elements, respectively, $K$ is the capital investment in the electronic information industry, $L$ is the labor input of the electronic information industry, and $Y$ represents the gross output value of the electronic information industry.

3.4. Summary of This Section. This section first introduces in detail the construction and optimization method of the agent behavior tree, as well as the characteristics of the agent behavior tree and the logical relationship represented by the common behavior tree, and optimizes the method. Then, through the construction of the early warning model of the SOM neural network, the flowchart 


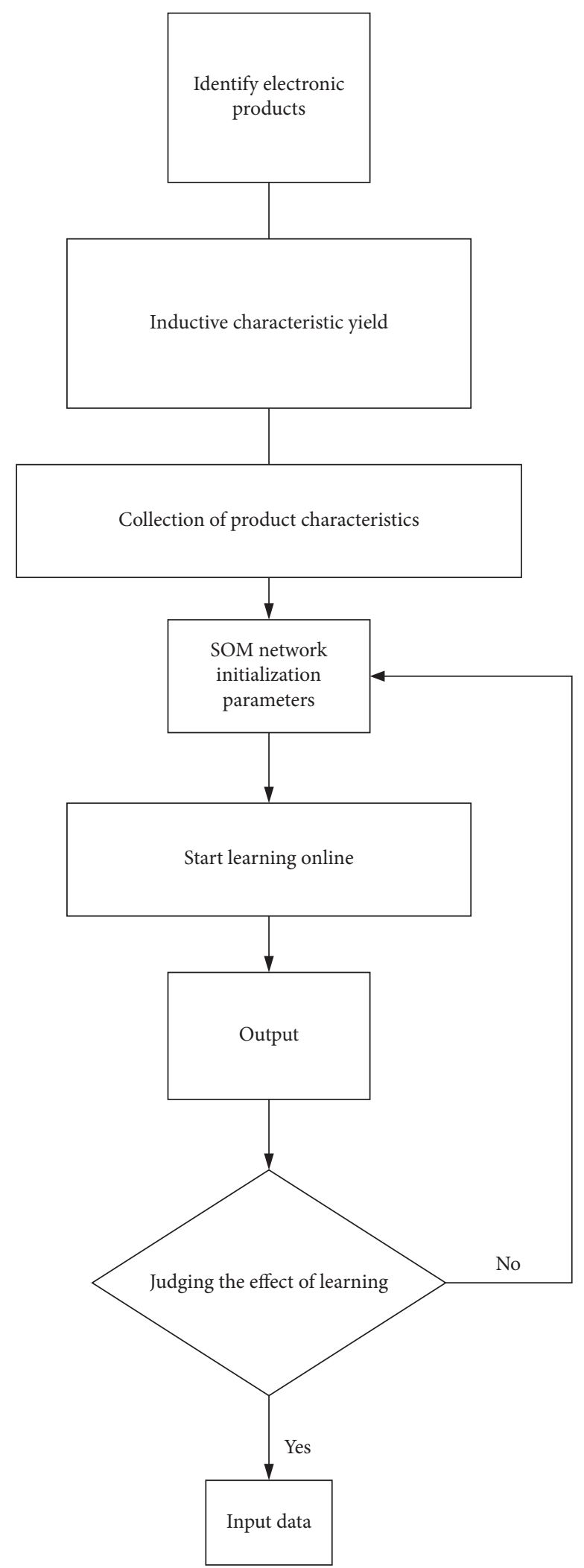

Figure 8: The structure flowchart of the SOM network early warning model.

of the early warning model of the SOM neural network is described, which is beneficial to the implementation of the sustainable development strategy. Finally, the construction of the production function model of the electronic information industry is proposed. The model analyzes the production factors of the electronic 


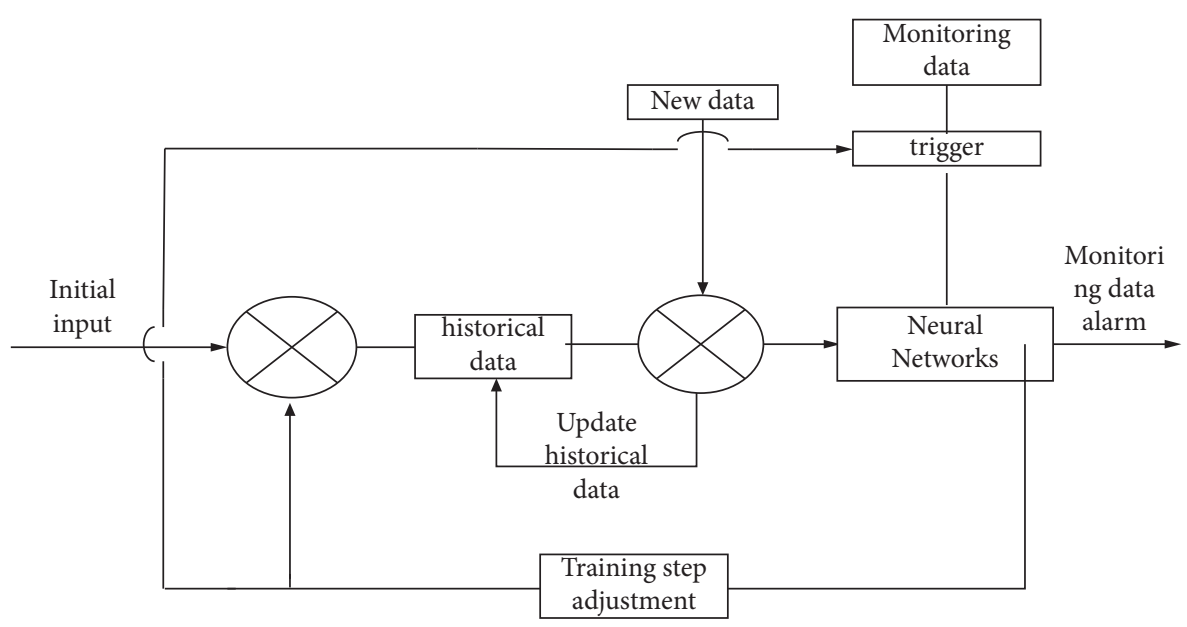

FIgURE 9: SOM neural network data update block diagram.

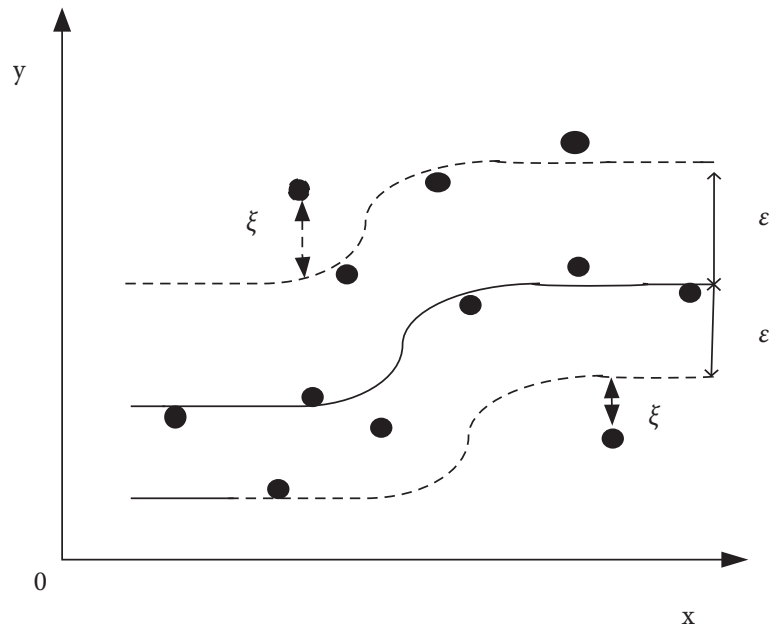

FIgURE 10: Schematic diagram of the basic idea of SVR.

information industry, then studies the influence of each factor on the development of the electronic information industry, and maximizes its application in electronic information engineering. The type is further proved.

\section{Predictive Model Experiment Analysis}

4.1. SVR Prediction Model Analysis and Optimization Advantages of SVR Model

(1) The model provides a strong theoretical basis, and the principle of structural risk minimization can be used.

(2) This model can effectively solve the problem of small samples and finally transform into a secondary planning problem.

(3) The topological structure of SVM is determined by the support vector. Because of the excessive dependence of the user's repeated attempts on the network topology, the traditional neural network is hidden.
In order to achieve the purpose of dispersing the two types of samples, we use SVM regression fitting to construct the optimal classification surface to minimize the distance between all training samples and the classification surface. The basic idea of SVR is shown in Figure 10.

4.2. Neural Network Early Warning and Forecasting Model. According to the neural network model parameters in $\mathrm{Ta}$ bles 1 and 2, input the training samples into the network model to train the network.

The changing trend of fitness is shown in Figure 11. It can be seen from the figure that the value of fitness is getting smaller and smaller, indicating that it has better performance. The accuracy of forecasting is improved, and good forecasting effects can be obtained.

This article uses the combination of artificial intelligence Internet of Things technology and air quality electronic monitoring equipment to carry out real-time monitoring, early warning, and forecasting to prevent it from happening, to achieve the desired result. Through the construction of neural network models and the analysis of the results, the 
TABLe 1: Air quality forecast PSO model parameters.

\begin{tabular}{lccccc}
\hline Population size & Evolutionary algebra & Particle dimension & Target error & Learning rate & Maximum number of training \\
\hline 50 & 100 & 82 & 0.00001 & 0.1 & 100 \\
\hline
\end{tabular}

TABLe 2: Neural network model parameters.

\begin{tabular}{lccccc}
\hline Target error & Learning rate & Number of training & Frequency & Input layer to hidden layer & Hidden layer to output layer \\
\hline 0.00001 & 0.1 & 100 & 100 & Tansig & Purelin \\
\hline
\end{tabular}

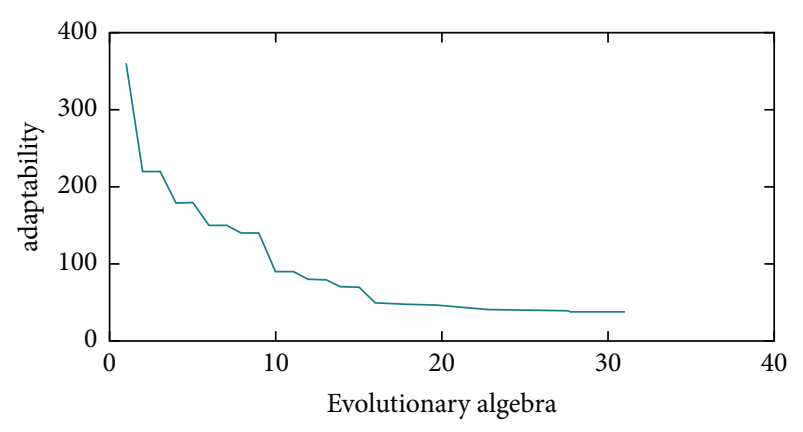

Figure 11: Trend of fitness.

monitoring revenue can be significantly improved under the monitoring of intelligent electronic equipment. The validity and superiority of the prediction determination through the neural network early warning forecast model are verified.

4.3. Summary of This Section. In this section, by constructing and optimizing the SVR prediction model, it is found that it avoids the traditional neural network's excessive dependence on the user's repeated trial and error of the network topology. In order to solve the problem of separating the two samples, an optimal classification surface is constructed. Through the analysis of the neural network early warning and forecasting model, AI is combined with the air quality electronic monitoring equipment to perform real-time monitoring, early warning, and forecasting. The validity and superiority of the prediction through the neural network early warning forecast model are verified. The discovery establishes the development direction for the application of electronic information.

\section{Conclusions}

With the improvement of the maturity of intelligent control applications, the artificial intelligence Internet of Things technology will inevitably further expand the research scope of intelligent control. Production control can be performed more finely and dynamically, and work efficiency can be significantly improved, which brings convenience to people's work and life and ultimately will forcefully promote the development of the national economy. However, the artificial intelligence Internet of Things technology is not mature enough and is still in an experimental stage. It provides a more reliable guarantee for the safety of personnel, reducing or avoiding the occurrence of tragedies. For this reason, the combination of artificial intelligence Internet of Things technology and electronic information engineering should be used scientifically and reasonably.

\section{Data Availability}

No data were used to support this study.

\section{Conflicts of Interest}

The authors declare that there are no conflicts of interest regarding the publication of this article.

\section{References}

[1] D. Hassabis, D. Kumaran, C. Summerfield, and M. Botvinick, "Neuroscience-inspired artificial intelligence," Neuron, vol. 95, no. 2, pp. 245-258, 2017.

[2] W. U. Zhao-Hui, "Research on the application of Internet of things technology to digital museum construction," Acta Geoscientia Sinica, vol. 38, no. 2, pp. 293-298, 2017.

[3] E. Ogidiaka, F. N. Ogwueleka, and M. Ekata Irhebhude, "Game-theoretic resource allocation algorithms for device-todevice communications in fifth generation cellular networks: a review," International Journal of Information Engineering and Electronic Business, vol. 13, no. 1, pp. 44-51, 2021.

[4] A. Bundy, "Preparing for the future of artificial intelligence," AI \& Society, vol. 32, no. 2, pp. 285-287, 2017.

[5] S. Jha and E. J. Topol, "Adapting to artificial intelligence," JAMA, vol. 316, no. 22, pp. 2353-2354, 2016.

[6] L. D. Raedt, K. Kersting, S. Natarajan, and D. Poole, "Statistical relational artificial intelligence: logic, probability, and computation," Synthesis Lectures on Artificial Intelligence and Machine Learning, vol. 10, no. 2, pp. 1-189, 2016.

[7] F. Goyache, J. J. Del Coz, J. R. Quevedo et al., "Using artificial intelligence to design and implement a morphological assessment system in beef cattle," Animal Science, vol. 73, no. 1, pp. 49-60, 2016.

[8] L. Rongpeng, Z. Zhao, X. Zhou et al., "Intelligent 5G: when cellular networks meet artificial intelligence," IEEE Wireless Communications, vol. 24, no. 5, pp. 175-183, 2017.

[9] P. Glauner, J. A. Meira, P. Valtchev, R. State, and F. Bettinger, "The challenge of non-technical loss detection using artificial intelligence: a survey," International Journal of Computational Intelligence Systems, vol. 10, no. 1, pp. 760-775, 2017.

[10] M. Seyedmahmoudian, B. Horan, T. K. Soon et al., "State of the art artificial intelligence-based MPPT techniques for mitigating partial shading effects on PV systems-a review," Renewable and Sustainable Energy Reviews, vol. 64, no. oct, pp. 435-455, 2016.

[11] Z. Wang and R. S. Srinivasan, "A review of artificial intelligence based building energy use prediction: contrasting the 
capabilities of single and ensemble prediction models," Renewable and Sustainable Energy Reviews, vol. 75, no. AUG, pp. 796-808, 2016.

[12] L. Caviglione, M. Gaggero, J. F. Lalande, W. Mazurczyk, and M. Urbanski, "Seeing the unseen: revealing mobile malware hidden communications via energy consumption and artificial intelligence," IEEE Transactions on Information Forensics and Security, vol. 11, no. 4, pp. 799-810, 2017.

[13] D. D. Miller and E. W. Brown, "Artificial intelligence in medical practice: the question to the answer," The American Journal of Medicine, vol. 131, no. 2, pp. 129-133, 2018.

[14] C. Cath, S. Wachter, B. Mittelstadt, M. Taddeo, and L. Floridi, "Artificial intelligence and the "good society": the US, EU, and UK approach," Science and Engineering Ethics, vol. 24, no. 7625, pp. 1-24, 2017.

[15] A. F. Chen, A. C. Zoga, and A. R. Vaccaro, "Point/counterpoint: artificial intelligence in healthcare," Healthcare Transformation, vol. 2, no. 2, pp. 84-92, 2017.

[16] T. Yang, A. A. Asanjan, E. Welles, X. Gao, S. Sorooshian, and $\mathrm{X}$. Liu, "Developing reservoir monthly inflow forecasts using artificial intelligence and climate phenomenon information," Water Resources Research, vol. 53, no. 4, pp. 2786-2812, 2017.

[17] M. Nasr, A. E. D. Mahmoud, M. Fawzy, and A. Radwan, "Artificial intelligence modeling of cadmium(II) biosorption using rice straw," Applied Water Science, vol. 7, no. 2, pp. 823-831, 2017.

[18] X. Wang, X. Li, and V. Leung, "Artificial intelligence-based techniques for emerging heterogeneous network: state of the arts, opportunities, and challenges," IEEE Access, vol. 3, no. 3, pp. 1379-1391, 2017.

[19] C. Modongo, J. G. Pasipanodya, B. T. Magazi et al., "Artificial intelligence and amikacin exposures predictive of outcomes in multidrug-resistant tuberculosis patients," Antimicrobial Agents and Chemotherapy, vol. 60, no. 10, pp. 5928-5932, 2016.

[20] Y. U. Bin and K. Kumbier, "Artificial intelligence and statistics," Frontiers of Information Technology \& Electronic Engineering, vol. 19, no. 1, pp. 6-9, 2018. 